\title{
Diagnosing COPD in non-smokers: splitting not lumping
}

\author{
Edwin R Chilvers, David A Lomas
}

Chronic obstructive pulmonary disease (COPD) is the diagnostic label given to patients with chronic airflow obstruction that is poorly reversible. ${ }^{1}$ As such, it is a non-specific disease entity and, like stroke or atrial fibrillation, can be the end result of multiple and often very differing conditions. A diagnosis of COPD based on spirometry alone should therefore trigger further investigation to identify the underlying cause and the application, if possible, of a more appropriate and specific diagnostic label. Hence while sarcoidosis, chronic asthma, organ-specific autoimmune disease, cystic fibrosis and multiple other pathologies can all cause COPD (and in a technical sense allow all such patients to be labelled as having COPD) most would recognise this as diagnostic duplicity.

Recent studies have highlighted the apparently high proportion of patients with COPD who have never smoked. Collectively, these studies suggest that even in developed countries cigarette smoking causes COPD in only $50-70 \%$ of patients. Indeed, the Swedish OLIN and US NHANES III studies reported that the population-attributable risk of COPD from smoking in these countries was $45 \%$ and $44 \%$, respectively. ${ }^{2} 3$ More recent studies in the UK suggest that $15 \%$ of adults above the age of 65 who have never smoked also have COPD. ${ }^{4}$ These data, however, do not gel with most UK physicians' practice, at least at a secondary care level. While there is little doubt that exposure to biomass smoke in developing countries and certain occupational insults in other settings can lead to COPD, ${ }^{6}$ clinical practice would suggest that the proportion of patients

Respiratory Medicine Division, Department of Medicine, University of Cambridge School of Clinical Medicine, Addenbrooke's Hospital, CUHNHSFT and Papworth Hospital NHS Foundation Trust, Cambridge, UK

Correspondence to Professor Edwin R Chilvers, Respiratory Medicine Division, Department of Medicine, University of Cambridge School of Clinical Medicine, Box 157, Level 5 Addenbrooke's Hospital, Hills Road, Cambridge CB2 000, UK; erc24@cam.ac.uk with genuine COPD in the UK who have never smoked is extremely small. Suggesting that COPD is common in never smokers living in developed countries (and hence by implication could be termed "idiopathic") runs the significant risk of leaving such patients underdiagnosed and inappropriately treated.

So what has led to the view that obstructive lung disease is common in nonsmokers? This matter has been reviewed recently by Salvi and Barnes ${ }^{7}$ who present mainly epidemiological studies, where a diagnosis of COPD has been made most commonly by questionnaire and spirometry alone. The problems associated with such studies are obvious and include poor case validation, failure to exclude patients with other common airway diseases such as asthma, bronchiectasis, tuberculosis and prior viral bronchiolitis, and insufficient scrutiny of patients' true exposure to tobacco smoke. These studies are further hampered by age-related changes in physiology; surely it is inappropriate to label an elderly patient with cough or breathlessness who has never smoked but who has mildly "obstructive" spirometry as having COPD? This matter was recently highlighted in a study by Hardie and colleagues who found that the Global Initiative for Obstructive Lung Disease (GOLD) guidelines for diagnosing and treating COPD falsely classify a substantial number of healthy, asymptomatic, never-smoking older adults as having COPD. Indeed, in their study, undertaken in residents of Bergen, Norway, $>50 \%$ of such individuals aged over 80 years would have been classified as having COPD. ${ }^{8}$ This conclusion is supported by a number of other studies conducted in primary care that highlight the pitfalls of using the current recommended spirometric criteria for COPD in the elderly. ${ }^{9}$ Hence we must avoid using spirometric data in such an absolute way as, say, a diabetologist might when using a blood sugar level to define diabetes and avoid making a diagnosis of COPD in all those with chronic airflow obstruction. This view does not, of course, undermine the importance of certain events such as poor socioeconomic status, low birth weight, concurrent asthma, previous tuberculosis, etc. to operate as important sensitisers to the effects of cigarette smoke ${ }^{7}$; however this does not equate with independent causation. Moreover, there seems to be little evidence to support the view that outdoor air pollution causes COPD.

Hence, while accepting that different phenotypes exist under the diagnostic label of "smoking-related COPD", this term would appear to be an entirely appropriate label for patients with a significant (eg, >5 pack year) smoking history, obstructive spirometry (postbronchodilator forced expiratory volume in $1 \mathrm{~s}\left(\mathrm{FEV}_{1}\right) /$ forced vital capacity (FVC) ratio $<70 \%$ ) and evidence of chronic bronchitis or emphysema; we would suggest that other causes of airflow obstruction must be sought in individuals in the UK who do not fulfil these criteria. This will avoid COPD becoming a "catch all" diagnostic label applied to multiple unrelated respiratory pathologies with clearly diverse aetiology. COPD would run the risk of becoming degraded into the "lung failure" equivalent of "heart failure" with little thought as to its true aetiology. The call to include non-smoking patients with "COPD" in trials designed to assess novel treatments ${ }^{7}$ is also likely to confound and confuse rather than add clarity. Cigarette smoking may be a less common cause of COPD in developing countries, but in the UK smoking should be considered a diagnostic prerequisite and its absence should trigger more detailed investigation. It is our view that the diagnostic process should start and not stop at the point of a spirometric diagnosis of airflow obstruction.

Acknowledgements The work in the author's laboratories is supported by the MRC, Wellcome Trust, EPSRC, Asthma-UK, BBSRC, GlaxoSmithKline and NIHR Cambridge Biomedical Research Centre

\section{Competing interests None.}

Provenance and peer review Commissioned; not externally peer reviewed.

Thorax 2010;65:465-466.

doi:10.1136/thx.2009.128421

\section{REFERENCES}

1. Rabe KF, Hurd S, Anzueto A, et al. Global initiative for chronic obstructive lung disease. Global strategy for the diagnosis, management, and prevention of chronic obstructive pulmonary disease: GOLD executive summary. Am J Respir Crit Care Med 2007; 176:532-55

2. Lundbäck $\mathbf{B}$, Lindberg $A$, Lindstrom $M$, et al Obstructive lung disease in Northern Sweden studies. 
Not 15 but $50 \%$ of smokers develop COPD? Report from the Obstructive Lung Disease in Northern Sweden Studies. Respir Med 2003;97:115-22.

3. Mannino DM, Buist AS, Petty TL, et al. Lung function and mortality in the United States: data from the first National Health and Nutrition Examination Survey follow up study. Thorax 2003;58:388-93.

4. Shahab L, Jarvis MJ, Britton J, et al. Prevalence, diagnosis and relation to tobacco dependence of chronic obstructive pulmonary disease in a nationally representative population sample. Thorax 2006:62:1043-47.

5. Torres-Duque C, Maldonado D, Pérez-Padilla R, et al. Forum of International Respiratory Studies (FIRS) Task Force on Health Effects of Biomass Exposure. Biomass fuels and respiratory diseases: a review of the evidence. Proc Am Thorac Soc 2008;5:577-90.

6. Blanc PD, Iribarren C, Trupin L, et al. Occupational exposures and the risk of COPD: dusty trades revisited. Thorax 2009:64:6-12.
7. Salvi SS, Barnes PJ. Chronic obstructive pulmonary disease in non-smokers. Lancet 2009;374: 733-43.

8. Hardie JA, Buist AS, Vollmer WM, et al. Risk of over-diagnosing COPD in asymptomatic never-smokers. Eur Respir J 2002:20: 1117-22.

9. Schermer TR, Smeele IJ, Thoonen BP, et al. Current clinical guideline definitions of airflow obstruction and COPD overdiagnosis in primary care. Eur Respir $\mathrm{J}$ 2008;32:945-52.

\section{Clinical networks for ILD: casting light on diffuse lung shadows}

\section{Nicholas K Harrison, ${ }^{1}$ Norman Johnson, ${ }^{2}$ Margaret Wilsher ${ }^{3}$}

The terms interstitial lung disease (ILD) and diffuse parenchymal lung disease are often used synonymously to refer to a disparate group of pulmonary disorders affecting the alveoli and/or respiratory bronchioles. Whilst many ILDs are rare disorders, as a group they account for $\sim 15 \%$ of the workload for an average respiratory physician. ${ }^{1}$

Despite this, and unlike diseases of the airway or lung cancer, the approach to diagnosis and management of ILD has not yet embraced multidisciplinary or shared, pathway-driven models of care. This, together with a paucity of treatments and the aggressive nature of some ILDs, casts a dim light on an already murky field where definitions are changing and even the pathogenesis remains unclear.

It is noteworthy that in idiopathic pulmonary fibrosis (IPF), the most common ILD, ${ }^{2}$ there have been no prospective randomised therapeutic trials undertaken in the UK for almost 20 years, and indeed there have only been two placebo-controlled randomised trials in this disease. ${ }^{3} 4$ However, there is no justification for this nihilism. Rather, the recent publication of the new BTS Guideline on ILD, published by the BTS in collaboration with the Thoracic Society of Australia and New Zealand and the Irish

\footnotetext{
${ }^{1}$ School of Medicine, Swansea University, Swansea, UK ; ${ }^{2}$ Whittington Hospital, London, UK; ${ }^{3}$ Department of Respiratory Medicine, Auckland City Hospital, New Zealand
}

Correspondence to Dr Nicholas K Harrison, School of Medicine, Swansea University, Swansea SA2 8PP, UK; Kim.Harrison@swansea-tr.wales.nhs.uk
Thoracic Society, is a timely reminder of our current state of knowledge, or perhaps ignorance, regarding diagnosis and management of ILDs. ${ }^{5}$ It is now appropriate to look beyond the Guideline and consider opportunities for improving care for patients with ILDs and in particular IPF. In the absence of promising new pharmaceuticals, such improvements can only be achieved through improvements to the organisation of care.

A study from Michigan examined the effect a multidisciplinary team (MDT), composed of specialist respiratory physicians, radiologists and pathologists, had on diagnosis of ILDs. ${ }^{6}$ It was found that whilst the addition of pathological information had the greatest impact on an individual MDT member's diagnostic confidence, this was consolidated when the team was permitted to reach a consensus. This provided support for the notion that surgical lung biopsy is no longer the diagnostic gold standard for ILD; but rather the new standard is consensus decision making. The same authors have demonstrated that diagnostic advice provided by MDTs in specialist (academic) centres differed significantly from that derived from community respiratory physicians. ${ }^{7}$ So, for the diagnosis of ILD, consensus opinions would appear to be more robust than those of individuals, and experts are more accurate than generalists.

As if to emphasise these conclusions, recent surveys examining clinical practice of respiratory physicians in both the UK and USA identified considerable variance in the way idiopathic interstitial pneumonias were diagnosed and treated. ${ }^{8} 9$ Whilst the Michigan data require validation in other healthcare settings and with other idiopathic interstitial pneumonias, they nonetheless raise serious concerns about diagnostic accuracy and hence appropriateness of decision making as currently practised by respiratory physicians, frequently in isolation and without expert opinion.

If reorganisation of the care pathway is required, which model will best suit the needs of patients and their physicians?

Patients with cystic fibrosis have been managed by a partnership of local and specialist centres for $>25$ years, although proof that such a configuration of service actually improves outcome took a long time to emerge. However, it is now widely accepted that a 'shared care' model, combining best local management with access to specialist centres, prolongs survival and improves quality of life for people with this disease. ${ }^{10}$ The Greater Manchester Lung Fibrosis Consortium was established in the early 1990s-an era that pre-dated digital image transfer and telemedicine. Its remit was to offer the facilities of a dedicated ILD clinic with a multidisciplinary approach to a wider area of North-West England and North Wales. It is almost 10 years since this group reported a retrospective study suggesting their model of care improved survival for patients with IPF under the age of $60 .{ }^{11}$ An integrated regional and community services model has recently been described for the management of patients with lung cancer in the Greater Toronto area. ${ }^{12}$ In this model, clinical resources were deployed to restructure services along patient-centred lines to devise a non-hierarchical clinical network with improved access to the specialist lung cancer team.

We can draw from these models to develop a paradigm of clinical networks which deliver a comprehensive package of high quality diagnostic services and patient information together with clear 